\title{
Acoustic and language analysis of speech for suicidal ideation among US veterans
}

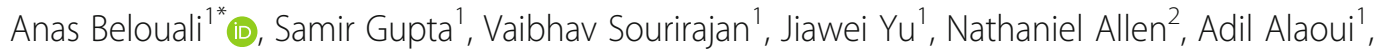
Mary Ann Dutton ${ }^{3}$ and Matthew J. Reinhard ${ }^{2,3}$

* Correspondence: ab873@
georgetown.edu
${ }^{1}$ Innovation Center for Biomedical
Informatics, Georgetown University
Medical Center, Washington, DC,
USA
Full list of author information is
available at the end of the article

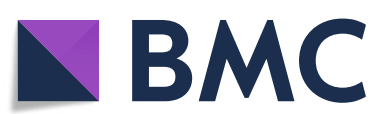

\begin{abstract}
Background: Screening for suicidal ideation in high-risk groups such as U.S. veterans is crucial for early detection and suicide prevention. Currently, screening is based on clinical interviews or self-report measures. Both approaches rely on subjects to disclose their suicidal thoughts. Innovative approaches are necessary to develop objective and clinically applicable assessments. Speech has been investigated as an objective marker to understand various mental states including suicidal ideation. In this work, we developed a machine learning and natural language processing classifier based on speech markers to screen for suicidal ideation in US veterans.

Methodology: Veterans submitted 588 narrative audio recordings via a mobile app in a real-life setting. In addition, participants completed self-report psychiatric scales and questionnaires. Recordings were analyzed to extract voice characteristics including prosodic, phonation, and glottal. The audios were also transcribed to extract textual features for linguistic analysis. We evaluated the acoustic and linguistic features using both statistical significance and ensemble feature selection. We also examined the performance of different machine learning algorithms on multiple combinations of features to classify suicidal and non-suicidal audios.

Results: A combined set of 15 acoustic and linguistic features of speech were identified by the ensemble feature selection. Random Forest classifier, using the selected set of features, correctly identified suicidal ideation in veterans with $86 \%$ sensitivity, 70\% specificity, and an area under the receiver operating characteristic curve (AUC) of $80 \%$.

Conclusions: Speech analysis of audios collected from veterans in everyday life settings using smartphones offers a promising approach for suicidal ideation detection. A machine learning classifier may eventually help clinicians identify and monitor high-risk veterans.
\end{abstract}

\section{Introduction}

Suicide prevention remains a challenging clinical issue, especially among Veterans. According to the most recent data from the United States Department of Veterans Affairs (VA), 17 veterans on average die from suicide per day and rates continue to rise [1]. After controlling for factors like age and gender, Veterans faced a 1.5 times greater risk

(c) The Author(s). 2021 Open Access This article is licensed under a Creative Commons Attribution 4.0 International License, which permits use, sharing, adaptation, distribution and reproduction in any medium or format, as long as you give appropriate credit to the original author(s) and the source, provide a link to the Creative Commons licence, and indicate if changes were made. The images or other third party material in this article are included in the article's Creative Commons licence, unless indicated otherwise in a credit line to the material. If material is not included in the article's Creative Commons licence and your intended use is not permitted by statutory regulation or exceeds the permitted use, you will need to obtain permission directly from the copyright holder. To view a copy of this licence, visit http://creativecommons.org/licenses/by/4.0/. The Creative Commons Public Domain Dedication waiver (http://creativecommons.org/publicdomain/zero/1.0/) applies to the data made available in this article, unless otherwise stated in a credit line to the data. 
for suicide compared to adult civilians. From 2005 to 2017, the suicide rate in the US civilian population increased $22.4 \%$, while rates among Veterans increased more than $49 \%$ [1]. To help address such alarming rates, there is an urgent need to develop objective and clinically applicable assessments for detecting high-risk individuals. Suicidal ideation is a known risk factor for suicide and has been found to be a predictor of immediate or long-term suicide attempts and deaths [2,3]. Screening high-risk groups such as veterans for suicidal thoughts is crucial for early detection and prevention [4].

To assess suicidality, healthcare providers use one of the several self-report screening tools such as the Suicidal Ideation Questionnaire (SIQ) or clinician-administered scales, such as the Ask Suicide-Screening Questions (ASQ) or the Columbia-Suicide Severity Rating Scale (C-SSRS) [5-7]. These traditional assessment measures have been found to have marginal predictive validity $[8,9]$. Another limitation of these assessments is that they require long visits with a clinician in order to establish rapport [10]. They also rely heavily on a subject's willingness to disclose their suicidal thoughts. Implicit bias may also affect the mental health assessment process and can lead to wrong screening results [11]. Due to these limitations, research into finding objective markers to aid clinical assessment is key in the fight against suicide.

Recent advances in digital technologies and mHealth devices have the potential to provide novel data streams for suicide prevention research [12]. Speech is an information-rich signal and measurable behavior that can be collected outside the clinical setting, which can increase accessibility to care and enable real-time and contextaware monitoring of an individual's mental state [13, 14]. Multiple studies have used voice characteristics as objective markers to understand and differentiate various mental states and psychiatric disorders [15]. These include investigations of voice in depression that identified many acoustic markers $[13,16,17]$. In another study, researchers were able to classify depressed and healthy speech using deep learning techniques applied to both audio and text features [18]. Research investigating speech and PTSD in US veterans identified 18 acoustic features and built a classifier to differentiate the 54 PTSD veterans from 77 controls with an area under the ROC curve of 0.95 [19]. A study of bipolar disease collected voice data from 28 patients using smartphones and classified affective states (manic vs depression episodes) longitudinally based on voice features with accuracy in the range of 0.61-0.74 [20]. There is a growing body of literature identifying linguistic patterns that express suicidal ideation [21, 22]. Different computational methods have been employed including feature extraction; topic modeling; word embeddings; traditional as well as deep learning methods to explore and classify suicidality in social media posts [21, 23-25]. Elevated use of absolutist words in tweets has been identified as a marker for anxiety, depression, and suicidal ideation [22]. Other work identified notable word clusters used in the Reddit SuicideWatch forum, which related to suicide risk factors including drug abuse (pills, medication, overdose); and depressive symptoms (pain, angry, sad) [26]. These reports and others support the feasibility and validity of detecting different mental disorders from speech using both acoustic and linguistic features.

Research on the spoken language of suicidal patients dates back as early as 1992, describing suicidal voices as sounding hollow, toneless, monotonous, with mechanical and repetitive phrasing, and a loss in intensity over an utterance [13, 27, 28]. It has been suggested that suicidal mental state causes changes to speech production mechanisms which 
in turn alter the acoustic properties of speech in measurable ways [28]. Comparisons of suicidal and non-suicidal speech in 16 adolescents identified glottal features as showing the strongest differences between the two groups. In particular, suicidal patients had lower Opening Quotient (OQ), and Normalized Amplitude Quotient (NAQ), acoustic measurements associated with more breathy voices [29]. Acoustic features such as fundamental frequency, amplitude modulation, pauses and rhythm-based features were also used to differentiate between suicidal and depressed patients $[17,30]$. Emotion recognition from natural phone conversations was used to classify 43 individuals with and without recent suicidal ideation with an AUC of 0.63 [31]. Similar work on phone conversations in 62 military couples predicted suicidal risk using multimodal features relating to behavior, emotion and turn-taking [32]. Recent work employed both linguistic and acoustic features of speech to classify 379 patients in one of three groups (suicidal, mentally ill but not suicidal, or controls) with accuracies in the range of $0.74-0.85$ [33, 34]. Although these are promising findings from different studies, they provide limited details on the acoustic and linguistic variables selected in the models.

Our work investigates speech features in 588 narrative audio diaries collected longitudinally from 124 US Veterans in a naturalistic setting using a mobile app that we developed for data collection. We conducted feature engineering on the recordings to extract sets of features and evaluated different classifiers and learning approaches. This study aims to identify and comprehensively characterize acoustic and linguistic features of speech that could classify suicidal ideation in veterans using audios collected in everyday life settings.

\section{Materials and methods}

\section{Study data and setting}

Data for this work was obtained as part of a larger intervention study for Gulf War Illnesses at the Washington DC VA Medical Center. One hundred forty-nine veterans meeting the Center for Disease Control's criteria for Gulf War Illness [35] were recruited and of these, 124 participants submitted 588 recordings via an Android smartphone app developed for data collection. The remaining 25 participants did not submit any recordings and were excluded from the analysis. An Android tablet (Samsung Galaxy Table 4) with the mobile app installed was provided to each veteran to enable participation from home.

All data was collected longitudinally from veterans in a naturalistic setting using the smartphone app. At each time-point of the study (week 0, week 4, week 8, 3 months, 6 months, 1 year), participants received reminder notifications and were prompted to complete multiple assessments, which included several self-report psychiatric scales and questionnaires. Veterans responded via audio recordings to open-ended questions about their general health in recent weeks/months and about their expectations from the study.

Each recorded response included a Patient Health Questionnaire (PHQ-9) administered as part of the health questionnaire battery. Item-9 of the PHQ-9 [36] is commonly used in research to screen for suicidal ideation and has been validated to be predictive of suicide in both the general population and in US veterans [37, 38]. It asks, "Over the last two weeks, how often have you been bothered by thoughts that you would be better off dead or of hurting yourself in some way?" Response options are "not at all", "several days", "more than half the days", or "nearly every day". We considered a subject as suicidal at the time of recording if they answered with any option other than "not at all". 


\section{Feature extraction and preprocessing}

Speech features can be divided into acoustic and linguistic features. We conducted comprehensive feature engineering on each recording to extract several sets of features. The study procedure is outlined in Fig. 1.

\section{Acoustic features}

A variety of acoustic parameter sets have been proposed for voice research and effective computing [39, 40]. Features including frequency; energy; amplitude; and MelFrequency Cepstral Coefficients (MFCC) have been used to classify several mental health states including suicidal ideation $[13,15,29,41]$. We extracted a total of 508 acoustic features from each recording using two audio signal analysis python libraries: pyAudioAnalysis [42]; and DisVoice [43]. Feature sets from both libraries have been previously used to classify psychiatric disorders and pathological speech [15, 44-46].

We used pyAudioAnalysis [42] to extract short-term feature sequences using a frame size of 50 milliseconds and a frame step of 25 milliseconds (50\% overlap). Then, we calculated recording level features as statistics on the short-term features (mean, maximum, minimum, median, standard deviation). The pyAudioAnalysis features include: zero crossing rate, energy and entropy of energy, chroma vector and deviation, spectral features composed of centroid, spread, entropy, flux, rolloff, and MFCCs. Using DisVoice, we computed prosodic features from continuous speech based on duration, fundamental frequency (F0), and energy. Phonation-based features were computed from sustained vowels and continuous speech utterances. For continuous speech, we computed the degree of unvoiced segments in addition to seven descriptors over voiced segments

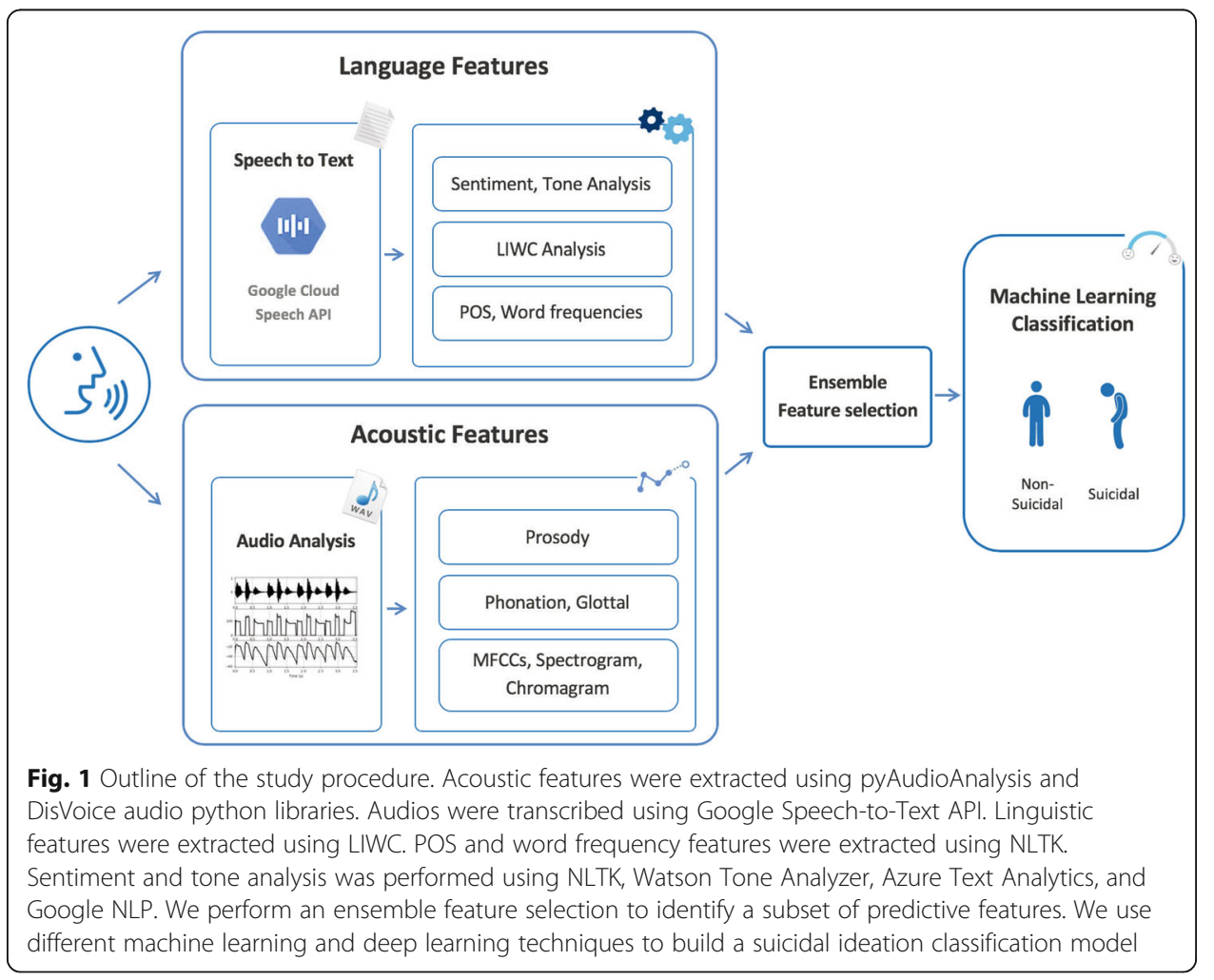


(first and second derivative of F0, jitter, shimmer, amplitude perturbation quotient, pitch perturbation quotient, logarithmic energy), then we derived higher-order statistics for each recording (mean, std., skewness, kurtosis). From sustained vowels, we computed 9 glottal features including: variability of time between consecutive glottal closure instants (GCI); average and variability of opening quotient (OQ) for consecutive glottal cycles; average and variability of normalized amplitude quotient (NAQ) for consecutive glottal cycles; average and variability of $\mathrm{H} 1 \mathrm{H} 2$ : difference between the first two harmonics of the glottal flow signal; and average and variability of Harmonic richness factor (HRF). In addition, four higher-order statistics were derived (mean, std., skewness, kurtosis).

\section{Linguistic features}

All audio files were automatically transcribed using the Google speech-to-text API, that achieves above 95\% accuracy in speech recognition tasks [47]. We manually verified transcriptions for $10 \%$ of the audios and while there were a few errors in the transcriptions, there were no major errors that changed the meaning of the answers. We did not correct the transcribed text corpus manually, as one of our goals was to assess the feasibility of an automated approach of both acoustic and linguistic analysis of speech. Subsequently, we used the transcribed text and various Natural Language Processing (NLP) techniques to extract different sets of textual features.

Parts of speech (POS) We use the NLTK library [48] to compute POS frequencies in the transcribed text. POS counts include word classes and lexical categories. Furthermore, we computed word frequencies of absolutist terms which have been associated with suicidal ideation in previous research [22].

Sentiment analysis Given the psychological nature of suicidal ideation, assessing the general polarity and emotions of the recordings is necessary. We compute sentiment scores and emotion level scores to detect joy, fear, sadness, anger, analytical, confident, and tentative tones in the language used by veterans. Sentiment analysis was performed using the following tools and APIs: NLTK, IBM Watson Tone Analyzer, Azure Text Analytics, and Google NLP. Most sentiment analysis tools are developed using text from reviews and tweets, which are different from transcribed text of audio recordings to openended questions about veterans' general health. Hence, we didn't limit our feature extraction to a single tool. We aim to obtain a better estimate of the valence and emotions through feature selection and weighting of the combination of sentiment features.

Linguistic Inquiry and Word Count program (LIWC) The LIWC software [49] is a text analysis tool that has been extensively used in the mental health space to explore various text corpora for hidden insights from linguistic patterns. The program produced 94 features per recording, based on validated dictionaries covering a wide range of categories to assess different psychological, affective, and linguistic properties.

Text visualization We used Scattertext [50], a text visualization tool to understand differences in speech between suicidal and non-suicidal veterans. The tool uses a scaled fscore, which takes into account the category-specific precision and term frequency. 
While a term may appear frequently in both groups, the scaled f-score determines if the term is more characteristic of one category versus another. Stopwords such as "the", "a", "an", "in" were excluded from the corpus.

\section{Statistical analysis}

We computed a total of 679 acoustic and linguistic features to understand speech in veterans with suicidal ideation. To compare suicidal and non-suicidal speech, we investigated these features by checking their statistical significance and magnitude of effect size. We used chi-square test for categorical variables and kruskal-wallis $H$-test for both continuous and ordinal variables. Raw $p$-values ( $p$-raw) were adjusted for multiple testing using the Bonferroni correction where $p$-adj $=p$-raw $\mathrm{x} \mathrm{n}$, where $\mathrm{n}$ is the number of independent tests. We define statistical significance as $p$-adj $<0.05$. We calculated the effect size using epsilon-squared $\left(\epsilon^{2}\right)$ to understand the influence of individual variables $[51,52]$. The goal of this first analysis is to infer any significant relationships between the characteristics of speech and suicidal ideation.

\section{Machine learning model development}

We performed a second analysis on the extracted feature set using Machine Learning (ML). ML is an analytical approach that can uncover hidden and complex patterns to help generate actionable predictions in clinical settings [53]. An essential step of any ML procedure is feature selection to reduce redundant variables and identify a stable subset of features. This can help create models that are easier to interpret and implement in real-life settings. We implemented an ensemble feature selection approach to select the top performing features across multiple selectors. This approach is known to improve the robustness of the selection process, especially in cases of high-dimensional and low sample size [54]. In particular, we used algorithms with built-in feature importance or coefficients such as ridge, lasso, random forest, and recursive feature elimination using logistic regression. For each algorithm, the best subset of features is selected and scores are assigned to each single feature. A mean-based aggregation is used to combine the results and calculate a mean-score for every feature. The meanscore provides a ranking of the top important and stable features. We used this score to retain different subsets of features using different thresholds. We evaluated model performance based on features with a ranking above the threshold. The best model performances used the top 15 ranked features. .

We observed class imbalance in our dataset with 1 suicidal recording for every 6 non-suicidal. To computationally deal with this imbalance, we used the SMOTE technique [55] to oversample the minority class in the training sets after partitioning the data during the learning process. It is essential to oversample after data partitioning to keep the test data representative of the original distribution of the dataset and avoid information leakage that can lead to overly optimistic prediction results [56].

We investigated six different supervised classification algorithms on the selected features and evaluated the results. The algorithms were: logistic regression (LR); random forest (RF); support vector machines (SVM); XGBoost (XGB); k-nearest neighbors $(\mathrm{KNN})$; and deep neural network (DNN). Prediction performance was assessed using the area under the receiver operating characteristic curve (AUC), which indicates how 
capable a model is at distinguishing between classes. Although this metric can be optimistic for imbalanced datasets, it still shows a relative change with better performing models, especially when higher sensitivity is desired (i.e., detection of suicidal recordings is more important) [57]. Additionally, we report sensitivity, specificity, F1 score, positive predictive value (PPV), negative predictive value (NPV), and accuracy. Since our data is imbalanced, it was important to assess the performance of the models based on all the metrics jointly. We used the Youden index [58] to identify the optimized prediction threshold to balance sensitivity and specificity.

For model evaluation and selection, we performed a nested cross-validation (CV) learning approach where we split the data into a 5-fold inner and a 5-fold outer CV sets. During each iteration of the nested CV, we kept 1 outer fold for testing (20\% of the samples) and used the 4 remaining folds in the 5-fold inner CV to search for the optimal model. We used a grid-search method in the inner loop to tune the different classification algorithms across a wide range of their respective hyperparameter settings. The final generalization error was estimated by averaging AUC scores over the outer test splits. We used nested CV, as opposed to regular k-fold CV, to reduce overfitting and produce stable and unbiased performance estimates that can generalize to unseen data [59-61].

The data partitioning applied during the nested CV was stratified. This means that each fold of the CV split had the same class distribution as the original dataset (1:6 ratio). Further, given the longitudinal aspect of the dataset, multiple recordings can belong to the same participant and may have different suicidal ideation labels across time. This potentially introduces data leakage where recordings from the same participant end up in both training and test folds. Since our goal was to build participantindependent models, we conducted a subject-wise CV to mirror the clinical use-case scenario of screening in newly recruited subjects [62]. Subject-wise CV ensures that recordings from the same patient will not appear in two different folds.

We built 3 different models to assess the predictive performance of acoustic and linguistic features separately and also when combined. All variables were normalized to a range of zero to one before fitting the models. The recordings were considered independent of the type of question asked or when they were recorded. In addition, we evaluated different minimum word counts and minimum audio length cutoffs for the inclusion of the recordings in the modeling.

\section{Results}

\section{Demographics and recordings characteristics}

Between May 2016 and January 2020, 124 participants submitted 588 recordings via the data collection mobile app. The average age of this group was 52.4 years $(\operatorname{std}=9.4)$ and the majority of participants were male veterans (79\%).

Table 1. Presents a breakdown of audio recordings by suicidality at each time-point of the study. Out of 588 audios, 504 were non-suicidal and 84 suicidal. All veterans recorded audios at least once, 27 veterans $(21.7 \%)$ recorded a maximum of eight recordings, and 74 veterans (59.6\%) recorded at least 4 recordings. During week 0 and week 8 , participants were asked to record two separate audios. Suicidal audios prevalence by time-point ranged from $10.8 \%$ at baseline to $23.6 \%$ at 3 months. Out of 124 patients 34 
(25.8\%) were suicidal at some point of the study. Eight patients were suicidal at every time point when they submitted a recording. Twenty-six patients converted between suicidal and non-suicidal ideation. After transcribing the audios, 15 recordings had no text transcriptions (5 suicidal and 10 non-suicidal). These audios were then manually verified and eventually excluded from the study, as they were either empty or had short intelligible speech. Results of the statistical analysis on the acoustic and linguistic features are summarized in Table 2.

\section{Acoustic analysis}

Average audio length was $44.19 \mathrm{~s}(\mathrm{std}=52.27)$. There were no significant differences between suicidal and non-suicidal recordings in audio length, overall audio loudness, or duration of pauses.

Suicidal recordings were mainly different from non-suicidals in terms of energy. Suicidal speech had a lower standard deviation of energy contours for voiced segments $(p$-adj $<0.001$, $\epsilon^{2}=0.04$ ), a lower kurtosis $\left(p\right.$-adj<0.001, $\epsilon^{2}=0.06$ ), and a skewness closer to zero ( $p$-adj< $\left.0.001, \epsilon^{2}=0.05\right)$ which reflect respectively flatter, less bursty, and less animated voice.

Suicidal speech had lower voiced tilt $\left(p\right.$-adj $\left.=0.04, \epsilon^{2}=0.03\right)$ and less energy entropy $\left(p\right.$-adj $\left.=0.04, \epsilon^{2}=0.03\right)$ thus displaying less vocal energy and less abrupt changes. Suicidal speech also exhibited a lower standard deviation of delta MFCC11 ( $p$-adj=0.004, $\epsilon^{2}=0.03$ ), delta MFCC12 ( $p$-adj=0.004, $\epsilon^{2}=0.03$ ), and delta MFCC1 ( $p$-adj=0.05, $\left.\epsilon^{2}=0.03\right)$. The decrease in time derivatives (delta) of MFCC coefficients indicates a lack of variance of energy over time in suicidal speech which can be interpreted as dull and more monotonous voices. Additionally, suicidal veterans produced speech that was irregular in time and displayed high variability between consecutive GCIs $(p$-adj $=0.035$, $\left.\epsilon^{2}=0.03\right)$, which can be interpreted as breathier voices.

\section{Linguistic analysis}

Average word count was 70.96 words per recording $(\mathrm{std}=93.76)$ with an average of 15.05 words per sentence $(s t d=9.62)$. There were no significant differences between suicidal and non-suicidal recordings in word count or words per sentence. Suicidal participants used more possessive pronouns $\left(p\right.$-raw $\left.=0.005, \epsilon^{2}=0.005\right)$ and more superlative adverbs $(p$-raw $=0.005$, $\left.\epsilon^{2}=0.008\right)$. The analysis of the LIWC scaled scores showed that suicidal participants also used more family references (e.g., daughter, dad, aunt) ( -raw $\left.=0.014, \epsilon^{2}=0.010\right)$ and more family male references (e.g., boy, his, dad) $\left(p\right.$-raw $\left.<0.001, \epsilon^{2}=0.021\right)$. Non-suicidal recordings contained more agentic language (e.g., win, success, better) ( $p$-raw $\left.=0.035, \epsilon^{2}=0.007\right)$. There were

Table 1 Breakdown of audio recordings by suicidality at each time-point of the study

\begin{tabular}{lllll}
\hline Time-point & $\begin{array}{l}\text { Non-suicidal } \\
\boldsymbol{N = 5 0 4}\end{array}$ & $\begin{array}{l}\text { Suicidal } \\
\mathbf{N = 8 4}\end{array}$ & $\begin{array}{l}\text { Total audios } \\
\mathbf{N = 5 8 8}\end{array}$ & $\begin{array}{l}\text { Participants } \\
\mathbf{N = \mathbf { 1 2 4 }}\end{array}$ \\
\hline Week 0, n (\%) & $214(89.2)$ & $26(10.8)$ & 240 & 120 \\
2 weeks follow-up, n (\%) & $63(87.5)$ & $9(12.5)$ & 72 & 72 \\
8 weeks follow-up, n (\%) & $100(84.7)$ & $18(15.3)$ & 118 & 50 \\
3 months follow-up, n (\%) & $42(76.4)$ & $13(23.6)$ & 55 & 55 \\
6 months follow-up, n (\%) & $45(86.5)$ & $7(13.5)$ & 52 & 52 \\
1 year follow-up, n (\%) & $40(78.4)$ & $11(21.6)$ & 51 & 51 \\
\hline
\end{tabular}

Audios are labeled using item-9 of the PHQ-9 assessment completed at each time-point ${ }^{a}$ During week 0 and week 8 , participants recorded two audios 
Table 2 Summary statistics of significant features for suicidal and non-suicidal groups

\begin{tabular}{|c|c|c|c|c|c|}
\hline Variables & $\begin{array}{l}\text { Overall } \\
\text { mean }(s t d)\end{array}$ & $\begin{array}{l}\text { Non-suicidal } \\
\text { mean (std) }\end{array}$ & $\begin{array}{l}\text { Suicidal } \\
\text { mean (std) }\end{array}$ & $\begin{array}{l}\mathrm{p}- \\
\text { adj }\end{array}$ & $\epsilon^{2}$ \\
\hline Time between GCls - mean & $\begin{array}{l}0.0039 \\
(0.0009)\end{array}$ & $0.0038(0.0009)$ & $\begin{array}{l}0.0043 \\
(0.0011)\end{array}$ & 0.03 & 0.03 \\
\hline Time between GCls - std & $\begin{array}{l}0.0072 \\
(0.0028)\end{array}$ & $0.0074(0.0029)$ & $\begin{array}{l}0.0060 \\
(0.0019)\end{array}$ & 0.05 & 0.03 \\
\hline Energy contour for voiced segments - std & $\begin{array}{l}2.8089 \\
(0.9230)\end{array}$ & $2.8806(0.9270)$ & $\begin{array}{l}2.3614 \\
(0.7624)\end{array}$ & $\begin{array}{l}< \\
0.001\end{array}$ & 0.04 \\
\hline Energy contour for voiced segments - skewness & $\begin{array}{l}-0.6763 \\
(0.3286)\end{array}$ & $-0.7059(0.3288)$ & $\begin{array}{l}-0.4916 \\
(0.2613)\end{array}$ & $\begin{array}{l}< \\
0.001\end{array}$ & 0.05 \\
\hline Energy contour for voiced segments - kurtosis & $\begin{array}{l}0.0866 \\
(0.7950)\end{array}$ & $0.1583(0.8101)$ & $\begin{array}{l}-0.3609 \\
(0.5018)\end{array}$ & $\begin{array}{l}< \\
0.001\end{array}$ & 0.06 \\
\hline $\begin{array}{l}\text { Energy contour for voiced segments - average tilt } \\
\text { of a linear estimation }\end{array}$ & $\begin{array}{l}-34.9081 \\
(24.2386)\end{array}$ & $\begin{array}{l}-36.2156 \\
(24.4906)\end{array}$ & $\begin{array}{l}-26.7487 \\
(20.9610)\end{array}$ & 0.04 & 0.03 \\
\hline $\begin{array}{l}\text { Energy contour for voiced segments - average } \\
\text { MSE of a linear estimation }\end{array}$ & $\begin{array}{l}5.7945 \\
(3.4302)\end{array}$ & $6.0285(3.4929)$ & $\begin{array}{l}4.3348 \\
(2.5833)\end{array}$ & 0.01 & 0.03 \\
\hline Delta energy entropy - std & $\begin{array}{l}0.3577 \\
(0.0788)\end{array}$ & $0.3631(0.0759)$ & $\begin{array}{l}0.3240 \\
(0.0878)\end{array}$ & 0.04 & 0.03 \\
\hline Delta MFCC11 - std & $\begin{array}{l}0.1881 \\
(0.0172)\end{array}$ & $0.1893(0.0169)$ & $\begin{array}{l}0.1805 \\
(0.0169)\end{array}$ & $\begin{array}{l}< \\
0.001\end{array}$ & 0.04 \\
\hline Delta MFCC12 - std & $\begin{array}{l}0.1823 \\
(0.0154)\end{array}$ & $0.1832(0.0152)$ & $\begin{array}{l}0.1761 \\
(0.0154)\end{array}$ & 0.01 & 0.03 \\
\hline Delta MFCC1 - std & $\begin{array}{l}1.0386 \\
(0.2200)\end{array}$ & $1.0525(0.2112)$ & $\begin{array}{l}0.9515 \\
(0.2528)\end{array}$ & 0.05 & 0.03 \\
\hline
\end{tabular}

Abbreviations: GCI Glottal closure instants, MSE Mean squared error, std. Standard deviation, MFCC Mel-Frequency Cepstral Coefficients, $p$-adj Adjusted $p$-value

no significant differences between the two groups in sentiment scores or usage of negative or positive emotion words. After adjusting for multiple testing, no linguistic features were significant.

Scattertext analysis (Fig. 2) shows the top words used by both suicidal and non-suicidal veterans. Over 40 thousand words from the text corpus were analyzed to assign a scaled f-score to each word. Ranking words by f-score can help identify which terms are more characteristic of suicidal ideation versus non-suicidal ideation. Top words used by veterans experiencing suicidal thoughts were: "certainly"; "pills"; "real”; "knees"; "month"; "old"; "CPAP; "got"; "happened"; "stop"; "VA"; "certain"; "doctor"; "daily basis". Top words used by non-suicidal veterans were: "energy"; "little bit"; "areas'; "aware”; "following"; "function"; "trying"; "find"; "noticed"; "bit"; “improve"; "days"; "group"; "meditation".

\section{Selected features and prediction performance}

Table 3 presents 15 acoustic and linguistic features retained by the ensemble feature selection approach. These variables were used for the combined modeling (acoustic + linguistic) which yielded the best results. Out of the selected features, four were linguistic and assessed the use of superlative adverbs, possessive pronouns, proper nouns, and agentic language. The remaining features were acoustic and related to energy dynamics, MFCC, F0, and glottal flow measurements (i.e., OQ, NAQ).

We evaluated different word count (WC) cutoffs to identify the minimum utterances needed to better discriminate suicidal ideation in recordings. Classification performances increased as we increased the WC minimum. The best classification results were obtained for recordings with a minimum of 25 words. 


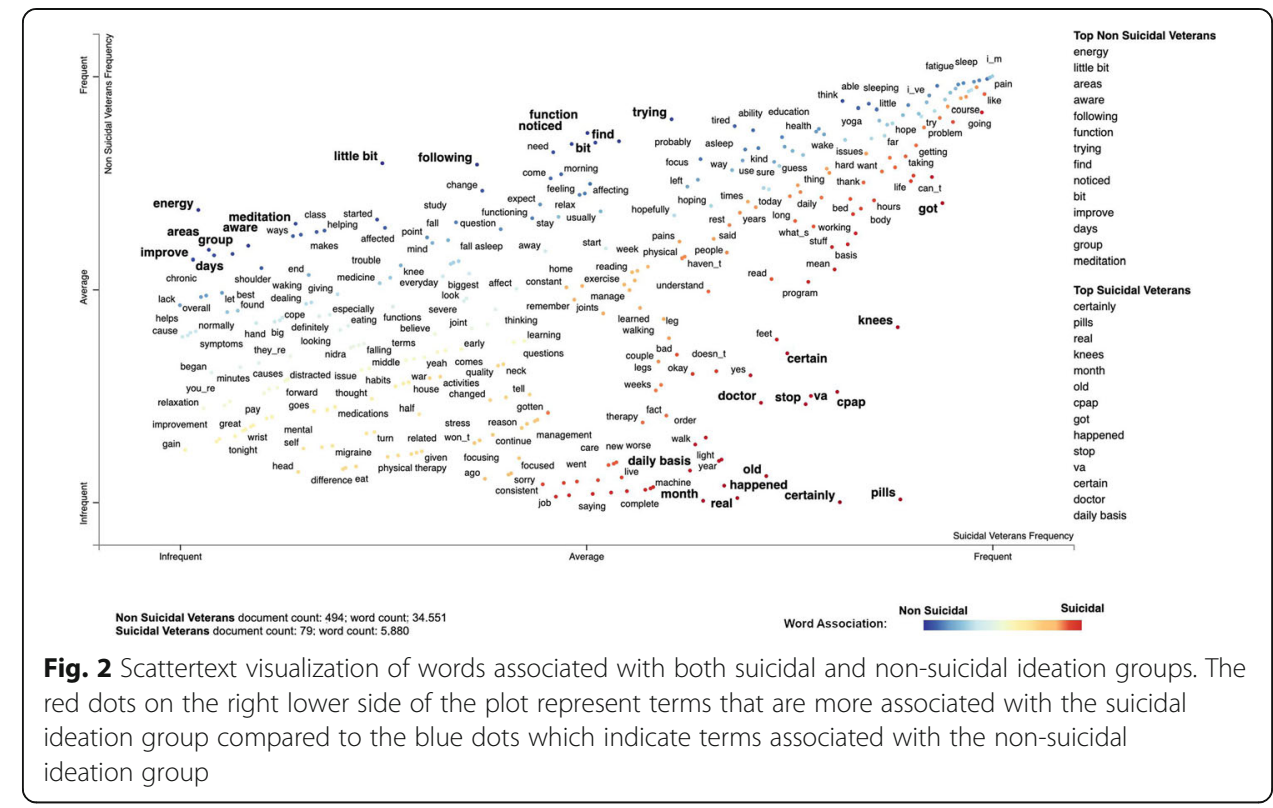

Table 4 presents the performance of the classifiers. The XGB classifier on acoustic features reached an overall AUC of $0.77(\operatorname{std}=0.08)$ with a sensitivity of $0.67(\operatorname{std}=0.18)$, specificity of $0.74(\operatorname{std}=0.21), F 1$ equal to $0.43(\operatorname{std}=0.12)$, and accuracy of $0.73(\operatorname{std}=$ 0.17). The LR classifier performed slightly better in terms of AUC with 0.78 ( $\operatorname{std}=0.12$ ) and sensitivity of $0.78(\mathrm{std}=0.11)$ but had a lower F1 equal to $0.38(\mathrm{std}=0.08)$, lower specificity of $0.64(\mathrm{std}=0.15)$, and a much lower accuracy of $0.66(\mathrm{std}=0.13)$.

On linguistic features, RF performed better than other classifiers overall with a sensitivity of $0.76(\operatorname{std}=0.09)$, specificity of $0.64(\operatorname{std}=0.17)$, accuracy of $0.65(\operatorname{std}=0.14), F 1$ equal to 0.38 ( $\mathrm{std}=0.09$ ), and an overall AUC of 0.74 ( $\mathrm{std}=0.07)$. XGB classifier performed better in terms of accuracy $=0.69(\mathrm{std}=0.13)$ and specificity $=0.70(\operatorname{std}=0.16)$ but performed lower on sensitivity $=0.67(\mathrm{std}=0.17), \mathrm{F} 1=0.37(\operatorname{std}=0.13)$, and $\mathrm{AUC}=$ $0.72($ std $=0.04)$.

Combining both acoustic and linguistic features improved most of the models in Table 4 . RF classifier correctly identified suicidal ideation in veterans with an overall sensitivity of 0.84 $(\mathrm{std}=0.09)$, specificity of $0.70(\mathrm{std}=0.16)$, accuracy of $0.72(\mathrm{std}=0.13)$, F1 equal to $0.45(\mathrm{std}=$ 0.09), and an AUC of 0.80 (std = 0.06). XGB performed better than RF in terms of F1 reaching a score of $0.47(\operatorname{std}=0.07)$, specificity of $0.79(\operatorname{std}=0.11)$, and accuracy of $0.78(\operatorname{std}=0.08)$. XGB performed much lower in sensitivity $0.74(\mathrm{std}=0.18)$ compared to RF. Overall, treebased models (RF and XGB) performed best on this dataset.

\section{Discussion}

In this study, we conducted a two-part analysis. First, we investigated the importance of the extracted acoustic and linguistic features using statistical significance. Second, we evaluated an ensemble feature selection approach to identify a subset of features that can be used in an ML model to detect suicidal ideation in veterans. The results demonstrate that characteristics of speech can be useful in differentiating between suicidal and non-suicidal recordings. Our findings also indicate that audios collected outside the clinical setting, using a mobile app, can be used to classify suicidal ideation 
Table 3 Description of the top 15 acoustic and linguistic features (rank-ordered by importance) retained for the combined machine learning modeling

\begin{tabular}{|c|c|c|}
\hline Feature & Type & Description \\
\hline $\begin{array}{l}\text { Delta energy } \\
\text { entropy (max) }\end{array}$ & $\begin{array}{l}\text { Acoustic } \\
\text { (Prosody) }\end{array}$ & Entropy of energy can be interpreted as a measure of abrupt changes. \\
\hline $\begin{array}{l}\text { Delta energy } \\
\text { (mean) }\end{array}$ & $\begin{array}{l}\text { Acoustic } \\
\text { (Prosody) }\end{array}$ & $\begin{array}{l}\text { Dynamic changes in energy relate to the perceptual characteristics of } \\
\text { pitch and loudness. }\end{array}$ \\
\hline $\begin{array}{l}\text { Energy contour } \\
\text { (kurtosis) }\end{array}$ & $\begin{array}{l}\text { Acoustic } \\
\text { (Prosody) }\end{array}$ & The kurtosis of the energy contour for voiced segments. \\
\hline Delta F0 (std) & $\begin{array}{l}\text { Acoustic } \\
\text { (Prosody) }\end{array}$ & $\begin{array}{l}\text { First derivative of the fundamental Frequency. Reduced F0 can indicate } \\
\text { low pitch and a flatter voice. }\end{array}$ \\
\hline MFCC5 (max) & $\begin{array}{l}\text { Acoustic } \\
\text { (MFCC) }\end{array}$ & $\begin{array}{l}\text { 5th MFCC coefficient. It can describe vocal tract changes in voice spectral } \\
\text { energy. }\end{array}$ \\
\hline $\begin{array}{l}\text { Superlative } \\
\text { adverbs }\end{array}$ & $\begin{array}{l}\text { Linguistic } \\
\text { (POS) }\end{array}$ & Use of superlative adverbs (e.g., biggest, hardest, etc.) \\
\hline OQ (skewness) & $\begin{array}{l}\text { Acoustic } \\
\text { (Glottal) }\end{array}$ & $\begin{array}{l}\mathrm{OQ} \text { is a measurement of the glottal flow. It can differentiate between a } \\
\text { breathy and tense voice. }\end{array}$ \\
\hline $\begin{array}{l}\text { Achievement } \\
\text { language }\end{array}$ & $\begin{array}{l}\text { Linguistic } \\
\text { (LIWC) }\end{array}$ & $\begin{array}{l}\text { Use of agentic language as defined by the LIWC achievement dictionary } \\
\text { (words such as win, success, better) }\end{array}$ \\
\hline $\begin{array}{l}\text { Delta chroma } 2 \\
\text { (min) }\end{array}$ & $\begin{array}{l}\text { Acoustic } \\
\text { (Prosody) }\end{array}$ & $\begin{array}{l}\text { A representation of spectral energy. Chroma-based features are also re- } \\
\text { ferred to as "pitch class profiles". }\end{array}$ \\
\hline Proper nouns & $\begin{array}{l}\text { Linguistic } \\
\text { (POS) }\end{array}$ & Use of singular proper nouns. \\
\hline $\begin{array}{l}\text { Delta energy } \\
\text { (median) }\end{array}$ & $\begin{array}{l}\text { Acoustic } \\
\text { (Prosody) }\end{array}$ & $\begin{array}{l}\text { Dynamic changes in energy relate to the perceptual characteristics of } \\
\text { pitch and loudness. }\end{array}$ \\
\hline $\begin{array}{l}\text { Delta chroma } 1 \\
\text { (median) }\end{array}$ & $\begin{array}{l}\text { Acoustic } \\
\text { (Prosody) }\end{array}$ & $\begin{array}{l}\text { A representation of spectral energy. Chroma-based features are also re- } \\
\text { ferred to as "pitch class profiles". }\end{array}$ \\
\hline $\begin{array}{l}\text { Delta MFCC5 } \\
\text { (max) }\end{array}$ & $\begin{array}{l}\text { Acoustic } \\
\text { (MFCC) }\end{array}$ & $\begin{array}{l}\text { The frame-based delta of the 5th MFCC coefficient. It can measure vocal } \\
\text { tract changes. }\end{array}$ \\
\hline NAQ (mean) & $\begin{array}{l}\text { Acoustic } \\
\text { (Glottal) }\end{array}$ & $\begin{array}{l}\text { Average NAQ is a measure of the glottal flow that can differentiate } \\
\text { between a breathy and tense voice. }\end{array}$ \\
\hline $\begin{array}{l}\text { Possessive } \\
\text { pronouns }\end{array}$ & $\begin{array}{l}\text { Linguistic } \\
\text { (POS) }\end{array}$ & Use of possessive pronouns (e.g., my, his, hers) \\
\hline
\end{tabular}

Abbreviations: FO Fundamental frequency, NAQ Normalized Amplitude Quotient, OQ Opening Quotient, std. Standard deviation, MFCC Mel-Frequency Cepstral Coefficients, POS Parts of speech

with an overall AUC of $0.80,86 \%$ sensitivity, and $70 \%$ specificity. This translates to a false positive rate (FPR) of $30 \%$ and a false negative rate (FNR) of $14 \%$. At baseline out of 120 patients (107 non-suicidal and 13 suicidal), the model is able to identify 11 suicidal patients out of 13 while misclassifying 32 non-suicidal patients as suicidal. An FPR of 30\% remains high but could be improved by targeting only veterans at high risk. Given that audio collection using an app is low cost, easy to administer, and safe, an improved model could be acceptable to both patients and practitioners if appropriate follow-up of individuals with positive screening is ensured. This is essential to avoid causing harm to inadvertently flagged veterans who may not benefit from an intervention.

In the first analysis, we sought to understand characteristics of suicidal speech in veterans and infer significant relationships. We identified 3 higher order statistics (std, kurtosis, skewness) related to energy contour of voiced segments. These variables displayed the largest effect size and indicated the strongest difference between the suicidal and non-suicidal groups. Veterans with suicidal ideation spoke in voices that were flatter, less bursty, and less animated. Additional energy-based variables such as speech tilt, 
Table 4 Classification results for suicidal ideation based on acoustic and linguistic features

\begin{tabular}{|c|c|c|c|c|c|c|c|c|}
\hline Feature set & Model & $\begin{array}{l}\text { Specificity } \\
\text { (std) }\end{array}$ & $\begin{array}{l}\text { Sensitivity } \\
\text { (std) }\end{array}$ & $\begin{array}{l}\text { PPV } \\
\text { (std) }\end{array}$ & $\begin{array}{l}\text { NPV } \\
\text { (std) }\end{array}$ & $\begin{array}{l}\text { Accuracy } \\
\text { (std) }\end{array}$ & $\begin{array}{l}\text { AUC } \\
\text { (std) }\end{array}$ & $\begin{array}{l}\text { F1 } \\
\text { (std) }\end{array}$ \\
\hline \multirow[t]{6}{*}{ Acoustic } & KNN & $0.52(0.23)$ & $0.78(0.13)$ & $\begin{array}{l}0.21 \\
(0.03)\end{array}$ & $\begin{array}{l}0.94 \\
(0.06)\end{array}$ & $\begin{array}{l}0.55 \\
(0.19)\end{array}$ & $\begin{array}{l}0.69 \\
(0.11)\end{array}$ & $\begin{array}{l}0.33 \\
(0.07)\end{array}$ \\
\hline & LR & $0.64(0.15)$ & $0.78(0.11)$ & $\begin{array}{l}0.26 \\
(0.02)\end{array}$ & $\begin{array}{l}0.95 \\
(0.05)\end{array}$ & $\begin{array}{l}0.66 \\
(0.13)\end{array}$ & $\begin{array}{l}0.78 \\
(0.12)\end{array}$ & $\begin{array}{l}0.38 \\
(0.08)\end{array}$ \\
\hline & $\mathrm{RF}$ & $0.64(0.12)$ & $0.76(0.17)$ & $\begin{array}{l}0.25 \\
(0.03)\end{array}$ & $\begin{array}{l}0.94 \\
(0.05)\end{array}$ & $\begin{array}{l}0.65 \\
(0.09)\end{array}$ & $\begin{array}{l}0.76 \\
(0.06)\end{array}$ & $\begin{array}{l}0.36 \\
(0.04)\end{array}$ \\
\hline & SVM & $0.54(0.30)$ & $0.74(0.18)$ & $\begin{array}{l}0.21 \\
(0.04)\end{array}$ & $\begin{array}{l}0.93 \\
(0.07)\end{array}$ & $\begin{array}{l}0.56 \\
(0.25)\end{array}$ & $\begin{array}{l}0.63 \\
(0.25)\end{array}$ & $\begin{array}{l}0.33 \\
(0.10)\end{array}$ \\
\hline & XGB & $0.74(0.21)$ & $0.67(0.18)$ & $\begin{array}{l}0.29 \\
(0.04)\end{array}$ & $\begin{array}{l}0.93 \\
(0.06)\end{array}$ & $\begin{array}{l}0.73 \\
(0.17)\end{array}$ & $\begin{array}{l}0.77 \\
(0.08)\end{array}$ & $\begin{array}{l}0.43 \\
(0.12)\end{array}$ \\
\hline & DNN & $0.70(0.06)$ & $0.66(0.20)$ & $\begin{array}{l}0.26 \\
(0.03)\end{array}$ & $\begin{array}{l}0.93 \\
(0.03)\end{array}$ & $\begin{array}{l}0.70 \\
(0.05)\end{array}$ & $\begin{array}{l}0.75 \\
(0.06)\end{array}$ & $\begin{array}{l}0.35 \\
(0.08)\end{array}$ \\
\hline \multirow[t]{6}{*}{ Linguistic } & KNN & $0.69(0.15)$ & $0.38(0.14)$ & $\begin{array}{l}0.16 \\
(0.03)\end{array}$ & $\begin{array}{l}0.87 \\
(0.05)\end{array}$ & $\begin{array}{l}0.65 \\
(0.11)\end{array}$ & $\begin{array}{l}0.52 \\
(0.07)\end{array}$ & $\begin{array}{l}0.22 \\
(0.03)\end{array}$ \\
\hline & LR & $0.57(0.24)$ & $0.66(0.20)$ & $\begin{array}{l}0.20 \\
(0.05)\end{array}$ & $\begin{array}{l}0.91 \\
(0.07)\end{array}$ & $\begin{array}{l}0.58 \\
(0.18)\end{array}$ & $\begin{array}{l}0.62 \\
(0.06)\end{array}$ & $\begin{array}{l}0.29 \\
(0.04)\end{array}$ \\
\hline & $\mathrm{RF}$ & $0.64(0.17)$ & $0.76(0.09)$ & $\begin{array}{l}0.26 \\
(0.09)\end{array}$ & $\begin{array}{l}0.94 \\
(0.08)\end{array}$ & $\begin{array}{l}0.65 \\
(0.14)\end{array}$ & $\begin{array}{l}0.74 \\
(0.07)\end{array}$ & $\begin{array}{l}0.38 \\
(0.09)\end{array}$ \\
\hline & SVM & $0.63(0.33)$ & $0.48(0.40)$ & $\begin{array}{l}0.13 \\
(0.09)\end{array}$ & $\begin{array}{l}0.88 \\
(0.08)\end{array}$ & $\begin{array}{l}0.61 \\
(0.24)\end{array}$ & $\begin{array}{l}0.49 \\
(0.15)\end{array}$ & $\begin{array}{l}0.19 \\
(0.14)\end{array}$ \\
\hline & XGB & $0.70(0.16)$ & $0.67(0.17)$ & $\begin{array}{l}0.27 \\
(0.08)\end{array}$ & $\begin{array}{l}0.93 \\
(0.05)\end{array}$ & $\begin{array}{l}0.69 \\
(0.13)\end{array}$ & $\begin{array}{l}0.72 \\
(0.04)\end{array}$ & $\begin{array}{l}0.37 \\
(0.06)\end{array}$ \\
\hline & DNN & $0.50(0.15)$ & $0.66(0.25)$ & $\begin{array}{l}0.16 \\
(0.04)\end{array}$ & $\begin{array}{l}0.90 \\
(0.06)\end{array}$ & $\begin{array}{l}0.52 \\
(0.11)\end{array}$ & $\begin{array}{l}0.63 \\
(0.14)\end{array}$ & $\begin{array}{l}0.25 \\
(0.07)\end{array}$ \\
\hline \multirow[t]{6}{*}{$\begin{array}{l}\text { Acoustic and } \\
\text { Linguistic }\end{array}$} & KNN & $0.61(0.22)$ & $0.78(0.22)$ & $\begin{array}{l}0.26 \\
(0.09)\end{array}$ & $\begin{array}{l}0.94 \\
(0.06)\end{array}$ & $\begin{array}{l}0.63 \\
(0.19)\end{array}$ & $\begin{array}{l}0.69 \\
(0.15)\end{array}$ & $\begin{array}{l}0.37 \\
(0.11)\end{array}$ \\
\hline & LR & $0.74(0.14)$ & $0.78(0.19)$ & $\begin{array}{l}0.35 \\
(0.14)\end{array}$ & $\begin{array}{l}0.95 \\
(0.05)\end{array}$ & $\begin{array}{l}0.75 \\
(0.11)\end{array}$ & $\begin{array}{l}0.77 \\
(0.12)\end{array}$ & $\begin{array}{l}0.46 \\
(0.13)\end{array}$ \\
\hline & $\mathrm{RF}$ & $0.70(0.16)$ & $0.84(0.09)$ & $\begin{array}{l}0.32 \\
(0.08)\end{array}$ & $\begin{array}{l}0.96 \\
(0.08)\end{array}$ & $\begin{array}{l}0.72 \\
(0.13)\end{array}$ & $\begin{array}{l}0.80 \\
(0.06)\end{array}$ & $\begin{array}{l}0.45 \\
(0.09)\end{array}$ \\
\hline & SVM & $0.86(0.13)$ & $0.52(0.32)$ & $\begin{array}{l}0.32 \\
(0.21)\end{array}$ & $\begin{array}{l}0.92 \\
(0.05)\end{array}$ & $\begin{array}{l}0.82 \\
(0.09)\end{array}$ & $\begin{array}{l}0.64 \\
(0.27)\end{array}$ & $\begin{array}{l}0.38 \\
(0.24)\end{array}$ \\
\hline & XGB & $0.79(0.11)$ & $0.74(0.18)$ & $\begin{array}{l}0.37 \\
(0.11)\end{array}$ & $\begin{array}{l}0.95 \\
(0.05)\end{array}$ & $\begin{array}{l}0.78 \\
(0.08)\end{array}$ & $\begin{array}{l}0.77 \\
(0.05)\end{array}$ & $\begin{array}{l}0.47 \\
(0.07)\end{array}$ \\
\hline & DNN & $0.68(0.06)$ & $0.70(0.15)$ & $\begin{array}{l}0.24 \\
(0.04)\end{array}$ & $\begin{array}{l}0.93 \\
(0.03)\end{array}$ & $\begin{array}{l}0.68 \\
(0.04)\end{array}$ & $\begin{array}{l}0.77 \\
(0.08)\end{array}$ & $\begin{array}{l}0.36 \\
(0.05)\end{array}$ \\
\hline
\end{tabular}

energy entropy, and MFCC coefficients, indicated that speech in suicidal veterans had less vocal energy, less abrupt changes, and was more monotonous. The analysis of the glottal flow parameters related to GCIs indicated a more breathy voice quality in suicidal veterans. These findings are in line with results from previous work on other risk groups. For example, a study that examined GCIs, OQ, and NAQ, found that suicidal adolescents had a more breathy voice quality when compared to non-suicidal adolescents [29]. In addition, multiple research studies used levels of energy dynamics and MFCC features to distinguish controls and depressed subjects who subsequently attempted suicide $[17,28,63]$. A recent study using crowd-sourced audios from the web also identified MFCC features and F0 as important voice biomarkers for suicidal ideation [64]. The general dullness of speech and reduction in energy has also been correlated with PTSD in veterans compared to controls [19]. 
The linguistic analysis, including sentiment analysis, produced no significant variables. This may be due to the fact that the sentiment tools we used are pre-trained on text corpora such as reviews or tweets, which is different from the transcribed text of the study. Nevertheless, we observed trends indicating more superlative adverbs, possessive pronouns, and proper nouns in suicidal speech. On the other hand, non-suicidal veterans used more agentic language based on the LIWC achievement score. The scatter text analysis, although exploratory in nature, provided frequently used words among non-suicidal and suicidal veterans. Overall, non-suicidal veterans used action verbs and words indicating improvements (e.g., function, improve, trying, find, noticed, aware). Whereas, we found that suicidal veterans spoke with certainty (e.g., certain, certainly), discussing topics such as chronic pain (pills, knees) or sleep problems (CPAP machine), when describing their general health in the past weeks and months. Interestingly, chronic pain and apnea have been linked to suicide as risk factors [65, 66]. Previous research on internet forums showed that suicidal subjects use more possessive pronouns and more absolutist words [22,67]. It is important to note that the frequently used terms identified in this analysis may come up in general assessment and be benign, and hence are not readily useful for assessment outside of technological approaches.

The second part of our analysis was building a classification model for suicidal ideation. The results show that acoustic-based models performed better $(\mathrm{AUC}=0.78)$ than models based on linguistic features alone $(\mathrm{AUC}=0.74)$. Given the links between suicidal ideation and language, we also explored advanced NLP techniques to improve the linguistic models, such as word and document embeddings. Classification using embeddings provided weak results (not presented), which was mainly due to the relatively small text corpus. Such techniques can be promising for the classification of suicidal ideation if applied to a much larger corpus. We achieved higher AUCs by combining both acoustic and linguistic features ( $A U C=0.80$ ). This is in line with previous research on depression and other mental states where fusion of different modalities such as audio, text, and visuals helped improve prediction results [18, 68-70]. AUCs reached by our models are comparable to previous research on suicidal ideation and speech in other risk groups, however, the few published studies either relied on smaller sample size or didn't discuss important features that went into the final prediction models [17, $29,33,71,72]$.

Most previous work investigating speech in suicidal ideation uses structured or semistructured clinical interviews, which may introduce interviewer biases [13]. In this study, we collect free speech recordings longitudinally from US veterans in a real-life setting using a mobile app. Collecting data digitally without the involvement of another person can reduce the stress associated with the fear of being judged and hence produce less biased recordings. Additionally, subjects using a mobile app might be willing to disclose more [73, 74]. Such an approach has the potential to be implemented for longitudinal and context-aware monitoring by collecting audio diaries from veterans at high risk. An application could be developed to collect audio diaries from veterans at high risk and share an assessment of suicidal ideation with a trained healthcare provider as a report. We acknowledge that as a standalone system, models could lead to a high rate of false positives. However, these systems do not aim to provide a diagnosis and are intended to support clinical decision making by augmenting the screening process. 
The impact of findings from this study may be limited by a number of factors. We relied on self-report to indicate whether a subject was suicidal or not at the time of the recording. Hence, it is possible that some of the recordings were mislabeled if a participant was not willing to divulge their suicidal ideation. Additionally, the simplification of suicidal ideation to binary labels, suicidal or not, does not consider that ideation might exist on a spectrum. Further, audios ranged from a few seconds to several minutes long and were made in a variety of everyday life settings which could have introduced background noise and quality issues. We believe that a quality check module could be developed within the data collection app and implemented separately during the audio recording step. This can help assess speech audibility and prompt subjects to re-record or move to a place that is quieter. An additional limitation may stem from possible confounders such as demographics (gender, sociolect, etc.) or other mental states that participants might suffer from such as depression; PTSD; or anxiety. This makes it difficult to determine whether the identified features are solely linked to suicidal ideation or might be linked to other comorbid mental states that are more likely to present in suicidal subjects. Future work conditioning on gender and assessing other mental states along with suicidal ideation could help improve the classifiers and further validate the identified features. Improvements to the classifiers could also come from different fusion methods of acoustic and linguistic features such as ensemble modeling or from context-based analysis where the questions asked are also weighted in the models.

\section{Conclusions}

We showed that speech analysis is a promising approach for detecting suicidal ideation in veterans. We also demonstrated that recordings collected longitudinally outside the clinical setting, using a mobile app, can be utilized for such analysis. Using predictive modeling, we identified a set of important acoustic and linguistic markers of speech that can be useful in classifying suicidal ideation in these recordings. The choice of the ML approach and dimensionality reduction techniques were important to optimize the performance of the classifiers and provide realistic estimations on unseen data. Further external validation and optimization are needed to validate and improve these findings. Overall, our work supports the feasibility of an automated approach of both acoustic and linguistic analysis of speech in everyday life settings, which holds the promise for real-time suicidal ideation assessment in high-risk veterans.

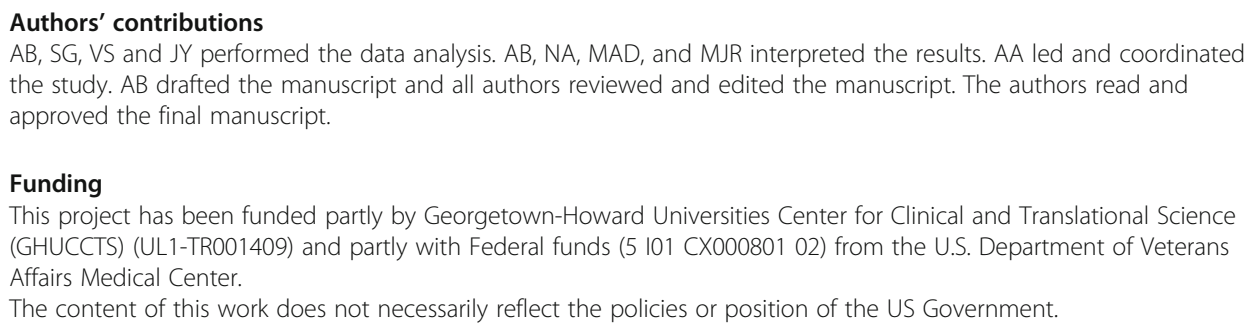

Availability of data and materials

The datasets generated during and/or analyzed during the current study are not publicly available due to content that potentially identifies participants. 


\section{Consent for publication}

All the authors listed have read and approved the manuscript.

\section{Competing interests}

The authors declare that they have no competing interests.

\section{Author details}

${ }^{1}$ Innovation Center for Biomedical Informatics, Georgetown University Medical Center, Washington, DC, USA. ${ }^{2}$ War Related IIIness and Injury Study Center, Veterans Affairs Medical Center, Washington, DC, USA. ${ }^{3}$ Department of Psychiatry, Georgetown University Medical Center, Washington, DC, USA.

Received: 18 September 2020 Accepted: 20 January 2021

Published online: 02 February 2021

\section{References}

1. Of Veterans Affairs D, Others. National veteran suicide prevention annual report. Washington: Department of Veterans Affairs; 2019.

2. Beck AT, Kovacs M, Weissman A. Assessment of suicidal intention: the Scale for Suicide Ideation. J Consult Clin Psychol. 1979;47:343-52.

3. Brown GK, Beck AT, Steer RA, Grisham JR. Risk factors for suicide in psychiatric outpatients: a 20-year prospective study. J Consult Clin Psychol. 2000;68:371-7.

4. Britton PC, Ilgen MA, Rudd MD, Conner KR. Warning signs for suicide within a week of healthcare contact in Veteran decedents. Psychiatry Res. 2012;200:395-9.

5. Reynolds WM. Suicidal ideation questionnaire (SIQ). Odessa: Psychological Assessment Resources; 1987. Available from: http://www.v-psyche.com/doc/Clinical\%20Test/Suicidal\%20ldeation\%20Questionnaire.doc

6. Horowitz LM, Bridge JA, Teach SJ, Ballard E, Klima J, Rosenstein DL, et al. Ask Suicide-Screening Questions (ASQ): a brief instrument for the pediatric emergency department. Arch Pediatr Adolesc Med. 2012;166:1170-6.

7. Posner K, Brent D, Lucas C, Gould M, Stanley B, Brown G, et al. Columbia-suicide severity rating scale (C-SSRS). New York: Columbia University Medical Center; 2008. Available from: https:/depts.washington.edu/ebpa/sites/default/files/C-SSRSLifetimeRecent-Clinical.pdf

8. Belsher BE, Smolenski DJ, Pruitt LD, Bush NE, Beech EH, Workman DE, et al. Prediction Models for Suicide Attempts and Deaths. JAMA Psychiatry. 2019:642. https://doi.org/10.1001/jamapsychiatry.2019.0174.

9. Franklin JC, Ribeiro JD, Fox KR, Bentley KH, Kleiman EM, Huang X, et al. Risk factors for suicidal thoughts and behaviors: A meta-analysis of 50 years of research. Psychol Bull. 2017;143:187-232.

10. Ganzini L, Denneson LM, Press N, Bair MJ, Helmer DA, Poat J, et al. Trust is the basis for effective suicide risk screening and assessment in veterans. J Gen Intern Med. 2013;28:1215-21.

11. Snowden LR. Bias in mental health assessment and intervention: theory and evidence. Am J Public Health. 2003;93:239-43.

12. Melia R, Francis K, Hickey E, Bogue J, Duggan J, O'Sullivan M, et al. Mobile Health Technology Interventions for Suicide Prevention: Systematic Review. JMIR mHealth and uHealth. 2020:e12516. Available from:. https://doi.org/10.2196/12516.

13. Cummins N, Scherer S, Krajewski J, Schnieder S, Epps J, Quatieri TF. A review of depression and suicide risk assessment using speech analysis. Speech Commun. 2015;71:10-49.

14. Johar S. Psychology of Voice. In: Johar S, editor. Emotion, Affect and Personality in Speech: The Bias of Language and Paralanguage. Cham: Springer International Publishing; 2016. p. 9-15.

15. Low DM, Bentley KH, Ghosh SS. Automated assessment of psychiatric disorders using speech: A systematic review. Laryngoscope Investig Otolaryngol. 2020;5:96-116.

16. Wang J, Zhang L, Liu T, Pan W, Hu B, Zhu T. Acoustic differences between healthy and depressed people: a crosssituation study. BMC Psychiatry. 2019;19:300.

17. France DJ, Shiavi RG, Silverman S, Silverman M, Wilkes DM. Acoustical properties of speech as indicators of depression and suicidal risk. IEEE Trans Biomed Eng. 2000;47:829-37.

18. Al Hanai T, Ghassemi MM, Glass JR. Detecting Depression with Audio/Text Sequence Modeling of Interviews. Interspeech; 2018. p. 1716-20.

19. Marmar CR, Brown AD, Qian M, Laska E, Siegel C, Li M, et al. Speech-based markers for posttraumatic stress disorder in US veterans. Depress Anxiety. 2019;36:607-16.

20. Faurholt-Jepsen M, Busk J, Frost M, Vinberg M, Christensen EM, Winther $\mathrm{O}$, et al. Voice analysis as an objective state marker in bipolar disorder. Transl Psychiatry. 2016;6:e856.

21. Sawhney R, Manchanda P, Singh R, Aggarwal S. A Computational Approach to Feature Extraction for Identification of Suicidal Ideation in Tweets. Proceedings of ACL 2018, Student Research Workshop. Melbourne, Australia: Association for Computational Linguistics; 2018. p. 91-8.

22. Al-Mosaiwi M, Johnstone T. In an Absolute State: Elevated Use of Absolutist Words Is a Marker Specific to Anxiety, Depression, and Suicidal Ideation. Clin Psychol Sci. 2018;6:529-42.

23. Sinha PP, Mishra R, Sawhney R, Mahata D, Shah RR, Liu H. \#suicidal - A Multipronged Approach to Identify and Explore Suicidal Ideation in Twitter. Proceedings of the 28th ACM International Conference on Information and Knowledge Management. New York: Association for Computing Machinery; 2019. p. 941-50.

24. Mishra R, Prakhar Sinha P, Sawhney R, Mahata D, Mathur P, Ratn SR. SNAP-BATNET: Cascading Author Profiling and Social Network Graphs for Suicide Ideation Detection on Social Media. Proceedings of the 2019 Conference of the North American Chapter of the Association for Computational Linguistics: Student Research Workshop. Minneapolis, Minnesota: Association for Computational Linguistics; 2019. p. 147-56.

25. Sawhney R, Manchanda P, Mathur P, Shah R, Singh R. Exploring and Learning Suicidal Ideation Connotations on Social Media with Deep Learning. Proceedings of the 9th Workshop on Computational Approaches to Subjectivity, Sentiment and Social Media Analysis. Brussels, Belgium: Association for Computational Linguistics; 2018. p. 167-75. 
26. Grant RN, Kucher D, León AM, Gemmell JF, Raicu DS, Fodeh SJ. Automatic extraction of informal topics from online suicidal ideation. BMC Bioinformatics. 2018;19:211.

27. Silverman SE. Method for detecting suicidal predisposition. US Patent. 1992 [cited 2020 Mar 20]. Available from: https:// patentimages.storage.googleapis.com/08/0e/27/15016f5fa2ae88/US5148483.pdf

28. Silverman SE, Ozdas A, Silverman MK. Method for analysis of vocal jitter for near-term suicidal risk assessment. US Patent. 2006 [cited 2020 Mar 20]. Available from: https:/patentimages.storage.googleapis.com/e8//f/25/ef4db4ef5cc7d6/US7139699.pdf

29. Scherer S, Pestian J, Morency L. Investigating the speech characteristics of suicidal adolescents. 2013 IEEE International Conference on Acoustics, Speech and Signal Processing; 2013. p. 709-13.

30. Hashim NW, Wilkes M, Salomon R, Meggs J. Analysis of timing pattern of speech as possible indicator for near-term suicidal risk and depression in male patients. International Proceedings of Computer Science and Information Technology, vol. 58: IACSIT Press; 2012. p. 6.

31. Gideon J, Schatten HT, MG MI, Provost EM. Emotion Recognition from Natural Phone Conversations in Individuals with and without Recent Suicidal Ideation. Interspeech; 2019. p. 3282-6.

32. Chakravarthula SN, Nasir M, Tseng S-Y, Li H, Park TJ, Baucom B, et al. Automatic Prediction of Suicidal Risk in Military Couples Using Multimodal Interaction Cues from Couples Conversations. ICASSP 2020 - 2020 IEEE International Conference on Acoustics, Speech and Signal Processing (ICASSP); 2020. Available from:. https://doi.org/10.1109/icassp40776.2020.9053246.

33. Pestian JP, Sorter M, Connolly B, Bretonnel Cohen K, McCullumsmith C, Gee J, et al. A Machine Learning Approach to Identifying the Thought Markers of Suicidal Subjects: A Prospective Multicenter Trial. Suicide Life Threat Behav. 2017;47:112-21.

34. Pestian JP, Grupp-Phelan J, Bretonnel Cohen K, Meyers G, Richey LA, Matykiewicz P, et al. A Controlled Trial Using Natural Language Processing to Examine the Language of Suicidal Adolescents in the Emergency Department. Suicide Life Threat Behav. 2016;46:154-9.

35. Fukuda K, Nisenbaum R, Stewart G, Thompson WW, Robin L, Washko RM, et al. Chronic multisymptom illness affecting Air Force veterans of the Gulf War. JAMA. 1998;280:981-8.

36. Kroenke K, Spitzer RL. The PHQ-9: a new depression diagnostic and severity measure. Psychiatr Ann. Slack Incorporated. 2002;32:509-15.

37. Louzon SA, Bossarte R, McCarthy JF, Katz IR. Does Suicidal Ideation as Measured by the PHQ-9 Predict Suicide Among VA Patients? PS. American Psychiatric Publishing. 2016;67:517-22.

38. Rossom RC, Coleman K, Ahmedani BK, Beck A, Johnson E, Oliver M, et al. Suicidal ideation reported on the PHQ9 and risk of suicidal behavior across age groups. J Affect Disord. 2017;215:77-84.

39. Eyben F, Scherer KR, Schuller BW, Sundberg J, André E, Busso C, et al. The Geneva Minimalistic Acoustic Parameter Set (GeMAPS) for Voice Research and Affective Computing. IEEE Transactions on Affective Computing. 2016;7:190-202.

40. Boersma, P. Praat : doing phonetics by computer. 2006. http://www.praat.org/ [cited 2020 Dec 4]; Available from: https://ci.nii.ac.jp/naid/10017594077/

41. Venek V, Scherer S, Morency L-P, Rizzo AS, Pestian J. Adolescent Suicidal Risk Assessment in Clinician-Patient Interaction. IEEE Transactions on Affective Computing; 2017. p. 204-15. Available from:: https://doi.org/10.1109/taffc.2016.2518665.

42. Giannakopoulos T. pyAudioAnalysis: An Open-Source Python Library for Audio Signal Analysis. PLoS One. 2015;10:e0144610.

43. Orozco-Arroyave JR, Vásquez-Correa JC, Vargas-Bonilla JF, Arora R, Dehak N, Nidadavolu PS, et al. NeuroSpeech: An open-source software for Parkinson's speech analysis. Digit Signal Process. 2018;77:207-21.

44. Vásquez-Correa JC, Orozco-Arroyave JR, Bocklet T, Nöth E. Towards an automatic evaluation of the dysarthria level of patients with Parkinson's disease. J Commun Disord. 2018;76:21-36.

45. Belalcázar-Bolaños EA, Orozco-Arroyave JR, Vargas-Bonilla JF, Haderlein T, Nöth E. Glottal Flow Patterns Analyses for Parkinson's Disease Detection: Acoustic and Nonlinear Approaches. Text, Speech, and Dialogue; 2016. p. 400-7. Available from: http://dx.doi.org/10.1007/978-3-319-45510-5_46

46. Vázquez-Romero A, Gallardo-Antolín A. Automatic Detection of Depression in Speech Using Ensemble Convolutional Neural Networks. Entropy. Multidisciplinary Digital Publishing Institute. 2020;22:688.

47. Cloud Speech-to-Text - Speech Recognition | Google Cloud [Internet]. Google Cloud. [cited 2020 Mar 26]. Available from: https://cloud.google.com/speech-to-text

48. Loper E, Bird S. NLTK: The Natural Language Toolkit. arXiv [cs.CL]. 2002. Available from: http://arxiv.org/abs/cs/0205028

49. Pennebaker JW, Booth RJ, Boyd RL, Francis ME. Linguistic Inquiry and Word Count: LIWC 2015 [Computer software]: Pennebaker Conglomerates. Inc; 2015.

50. Kessler JS. Scattertext: a Browser-Based Tool for Visualizing how Corpora Differ. arXiv [cs.CL]. 2017. Available from: http:// arxiv.org/abs/1703.00565

51. Tomczak M, Tomczak E. The need to report effect size estimates revisited. An overview of some recommended measures of effect size. Akademia Wychowania Fizycznego w Poznaniu; 2014; Available from: https://www.wbc.poznan. pl/dlibra/publication/413565/edition/325867?language $=$ pl

52. Rea LM, Parker RA. Designing and Conducting Survey Research: A Comprehensive Guide: John Wiley \& Sons; 2014.

53. Shah P, Kendall F, Khozin S, Goosen R, Hu J, Laramie J, et al. Artificial intelligence and machine learning in clinical development: a translational perspective. NPJ Digit Med. 2019;2:69.

54. Pes B. Ensemble feature selection for high-dimensional data: a stability analysis across multiple domains. Neural Comput Appl. 2019; Available from. https://doi.org/10.1007/s00521-019-04082-3.

55. Chawla NV, Bowyer KW, Hall LO, Kegelmeyer WP. SMOTE: Synthetic Minority Over-sampling Technique. arXiv [cs.Al]. 2011. Available from: http://arxiv.org/abs/1106.1813

56. Vandewiele G, Dehaene I, Kovács G, Sterckx L, Janssens O, Ongenae F, et al. Overly Optimistic Prediction Results on Imbalanced Data: Flaws and Benefits of Applying Over-sampling. arXiv [eess.SP]. 2020. Available from: http:/arxiv.org/abs/2001.06296

57. Pencina MJ, D'Agostino RB, Massaro JM. Understanding increments in model performance metrics. Lifetime Data Anal. 2013;19:202-18.

58. Fluss R, Faraggi D, Reiser B. Estimation of the Youden Index and its associated cutoff point. Biom J. 2005;47:458-72.

59. Vabalas A, Gowen E, Poliakoff E, Casson AJ. Machine learning algorithm validation with a limited sample size. PLoS One. 2019;14:e0224365.

60. Cawley GC, Talbot NLC. On Over-fitting in Model Selection and Subsequent Selection Bias in Performance Evaluation. J Mach Learn Res. 2010;11:2079-107. 
61. Dwyer DB, Falkai P, Koutsouleris N. Machine Learning Approaches for Clinical Psychology and Psychiatry. Annu Rev Clin Psychol. 2018;14:91-118.

62. Saeb S, Lonini L, Jayaraman A, Mohr DC, Kording KP. The need to approximate the use-case in clinical machine learning. Gigascience. 2017;6:1-9.

63. Cross Validation of Cepstral Coefficients in Classifying Suicidal Speech from Depressed Speech. International Institute of Engineers (IIE) May 22-23, 2015 Dubai (UAE). International Institute of Engineers; 2015. Available from: http://iieng.org/ siteadmin/upload/8111E0515057.pdf

64. Zhang L, Durvuri R, Chandra KKL, Nguyen T, Ghomi RH. Automated voice biomarkers for depression symptoms using an online cross-sectional data collection initiative. Depress Anxiety. 2020;37:657-69.

65. McCall WV, Black CG. The link between suicide and insomnia: theoretical mechanisms. Curr Psychiatry Rep. 2013;15:389.

66. Hassett AL, Aquino JK, Ilgen MA. The risk of suicide mortality in chronic pain patients. Curr Pain Headache Rep. 2014;18:436.

67. De Choudhury M, Kiciman E, Dredze M, Coppersmith G, Kumar M. Discovering Shifts to Suicidal Ideation from Mental Health Content in Social Media. Proc SIGCHI Conf Hum Factor Comput Syst. 2016;2016:2098-110.

68. D'Mello S, Kory J. Consistent but modest: a meta-analysis on unimodal and multimodal affect detection accuracies from 30 studies. Proceedings of the 14th ACM international conference on Multimodal interaction. New York: Association for Computing Machinery; 2012. p. 31-8.

69. Dham S, Sharma A, Dhall A. Depression Scale Recognition from Audio, Visual and Text Analysis. arXiv [cs.CV]. 2017. Available from: http://arxiv.org/abs/1709.05865

70. Jan A, Meng H, Gaus YFBA, Zhang F. Artificial Intelligent System for Automatic Depression Level Analysis Through Visual and Vocal Expressions. IEEE Transactions on Cognitive and Developmental Systems. 2018;10:668-80.

71. Silverman MK. Methods for evaluating near-term suicidal risk using vocal parameters. J Acoustical Soc Am. 2010:2259. Available from: https://doi.org/10.1121/1.3500785.

72. Keskinpala HK, Yingthawornsuk T, Wilkes DM, Shiavi RG, Salomon RM. Screening for high risk suicidal states using melcepstral coefficients and energy in frequency bands. 2007 15th European Signal Processing Conference; 2007. p. 2229-33.

73. Kretzschmar K, Tyroll H, Pavarini G, Manzini A, Singh I, Group NYPA. Can your phone be your therapist? Young people's ethical perspectives on the use of fully automated conversational agents (chatbots) in mental health support. Biomed Inform Insights. SAGE Publications Sage UK: London, England. 2019;11:1178222619829083.

74. Lucas GM, Gratch J, King A, Morency L-P. It's only a computer: Virtual humans increase willingness to disclose. Comput Human Behav. Elsevier. 2014;37:94-100.

\section{Publisher's Note}

Springer Nature remains neutral with regard to jurisdictional claims in published maps and institutional affiliations.

\section{Ready to submit your research? Choose BMC and benefit from:}

- fast, convenient online submission

- thorough peer review by experienced researchers in your field

- rapid publication on acceptance

- support for research data, including large and complex data types

- gold Open Access which fosters wider collaboration and increased citations

- maximum visibility for your research: over $100 \mathrm{M}$ website views per year

At $\mathrm{BMC}$, research is always in progress.

Learn more biomedcentral.com/submissions 